\title{
The use of Statistical Parametric Mapping (SPM96) as a decision aid in the differential diagnosis of dementia using $99 \mathrm{~m}$ Tc-HMPAO SPECT
}

\author{
A. Barnes ${ }^{\mathrm{a}}$, D. Lusman ${ }^{\mathrm{b}}$, J. Patterson ${ }^{\mathrm{a}}$, \\ D. Brown ${ }^{\mathrm{c}}$ and D. Wyper ${ }^{\mathrm{a}}$ \\ ${ }^{\mathrm{a}}$ Institute of Neurological Sciences, Southern General \\ Hospital, Glasgow, UK \\ ${ }^{\mathrm{b}}$ Garnock Day Hospital, Ayrshire Central Hospital, \\ Irvine, $U K$ \\ ${ }^{\mathrm{c}}$ Consultant Psychiatrist, (Room 5-7) Southbank \\ Centre, 1 Strathkelvin Place, Kirkintilloch, Glasgow \\ G66 1XQ, UK
}

In this study standard patterns of cerebral perfusion based on classifications described in the literature have been chosen and the ability of experienced imaging specialists to categorise the ${ }^{99 \mathrm{~m}}$ Tc HMPAO SPECT scans of patients referred to the department for investigation of dementia has been compared before and after the calculation of Statistical Parametric Maps (SPM - Wellcome Dept of Cognitive Neurology). The primary aim was to investigate whether SPM is an effective decision aid and whether it impacts on the confidence of image reporting. The secondary aim was to examine the influence of SPM on the agreement between image reporting and clinical diagnosis. The results showed that there was a slight decrease in agreement between the imaging specialists after the introduction of additional information from SPM ( $K=0.57$ to $K=0.5$ ) and that agreement between imaging reporting (including information from SPM) and clinical diagnosis was moderate $(K=0.28)$. This study was able to confirm that SPM is capable of producing meaningful significance maps of individual patients in a routine clinical environment. However, there was no overwhelming evidence that SPM was able to resolve many of the dilemmas associated with the use of SPECT for the differential diagnosis of dementia. In particular, interpretation of SPECT perfusion patterns in dementia is a bigger problem than the initial identification of abnormalities.

\section{Introduction}

Advances in medical imaging have continued to enhance its role in the diagnosis of many neurological conditions. At the same time, imaging specialists have been faced with greater demands on their expertise to interpret the increasingly complex threedimensional image data sets obtained from the various structural (CT and MRI) and functional (PET, SPECT and fMRI)imaging modalities. Efforts at exploiting the digital nature of the imaging data by utilising intelligent computational strategies such as neural networks to perform image interpretation have usually been unable to match the abilities of experienced radiologists. A more effective approach has been the development of decision support systems which can be used by the radiologist to aid image interpretation [49].

The simplest decision aid is a database of normal images which can be viewed and accessed in realtime [49]. The next level of complexity is automation of the process by which an individual patient scan is compared statistically to the normal database. The present study involves an assessment of the impact of one such system, Statistical Parametric Mapping (SPM), on the differential diagnosis of dementia using SPECT imaging of cerebral perfusion.

SPM was developed by Friston and colleagues [3, $11,12]$ for the analysis of functional neuroimaging data from PET. It performs an automated coregistration of all brain images to a standard stereotactic space, followed by a voxelwise statistical analysis based on the theory of Gaussian fields. The statistical analysis locates areas of significant change in the mean voxel intensity between groups of scans of different brain states and/or from different groups of subjects. SPM is ideally suited to the low resolution data provided by some SPECT systems and although it was not initially in- 
tended for routine clinical use the temptation to use this powerful technique in radiological diagnosis has recently become too strong to resist [43].

The differential diagnosis of dementia includes both reversible and non-progressive disorders [13] such as depression, delirium, metabolic disturbances as well as age-associated memory impairment. Clinicopathological studies have confirmed Alzheimer's disease (DAT) as the most common neurodegenerative cause of dementia [27] but other pathological categories such as frontotemporal dementia (FTD) [21] and Lewy body dementia (LBD) [30] account for a significant proportion of patients with memory and cognitive decline. In some countries, however, the most prevalent form of dementia is vascular dementia (DVaT) [38, 42]. Co-existing neuropathological findings are very common [24] and coexisting cerebrovascular disease certainly worsens the prognosis in DAT [17].

The importance of accurate differential diagnosis in dementia is no longer focused solely on the exclusion of reversible dementias or depression but is now crucial to the targeting of specific therapies as highlighted by recent experience with cholinesterase inhibitors as cognitive enhancers in mild and moderate DAT [7]. However, difficulties in making an accurate clinical diagnosis in everyday practice are well recognised and result in an accuracy of no better than 75\% [24]. Accepted clinical diagnostic criteria have recently been criticised on the basis of unacceptable negative predictive values as low as 33\% [34]. A definite diagnosis of Alzheimer type dementia remains within the domain of histopathological examination.

The basis for the use of SPECT in the differential diagnosis of dementia lies in the different function-related patterns of cerebral perfusion identified in each of the pathological categories described above $[14,15,18,22$, $44,45,48]$. SPECT images of probable Alzheimer's disease as defined by DSM-IV-R criteria [2] show regional deficits in the parieto-temporal cortex (sometimes asymmetrical) [23,33], those of frontotemporal dementia (FTD) show characteristic reductions in the anterior regions of the cortex including anterior aspects of the temporal lobe [32] while multi-focal lesions throughout the cortex are generally found in dementia of the vascular type (DVaT) $[33,48]$. Lewy Body Dementia (LBD) is associated with a SPECT pattern strikingly similar to that found in DAT [52] and its differentiation using perfusion imaging is therefore difficult. Studies in age-associated memory impairment confirm only minimal perfusion abnormalities [36] while those found in depression are confined mainly to the left dor- solateral pre-frontal region and are distinct from those found in neurodegenerative dementia [8].

In the present study standard patterns of cerebral perfusion based on the above classifications have been chosen and the ability of experienced imaging specialists to categorise the SPECT scans of patients referred to the department for investigation of dementia has been compared before and after the calculation of the SPM maps. The primary aim was therefore to investigate whether SPM is an effective decision aid and whether it impacts on the confidence of image reporting. As a secondary objective an attempt was made to examine its influence on the relationship between image reporting agreed by the two observers (imaging consensus) and clinical diagnosis agreed by the two community based psychiatrists (clinical consensus) at an average follow-up period of 25 months.

\section{Methods}

\subsection{Patients}

53 patients who had been referred to the Institute of Neurological Sciences by community based psychiatrists during the period April 1994 to December 1996 were recruited retrospectively for the purposes of this study. The patients were selected on the basis of imaging and clinical criteria as follows:

a) The SPECT image data set covered most of the cerebrum, with no evidence of patient movement during the scan. This requirement for a robust data set was necessary to ensure an optimal SPM analysis.

b) The referral request indicated that dementia was included within the differential diagnosis but that clinical criteria alone had failed to provide a confident diagnosis. This otherwise unselected group is likely to represent those patients who provide the greatest diagnostic challenge in a routine clinical setting.

Initial diagnostic investigations at the time of the ${ }^{99 \mathrm{~m}} \mathrm{Tc}$ - HMPAO SPECT scan included neuropsychological, neurological and in some cases radiological (CT or MRI) examinations. Follow-up or review of case notes was carried out by two psychiatrists at a mean period of 25 months from the SPECT scan (range 5-50). Of the 53 initially recruited 14 were excluded; the case notes were unavailable for 4 patients, 1 patient died within time for adequate follow-up (no post- 
mortem information), 6 patients declined participation and 3 were unobtainable at follow up. Of the remaining 39 cases (age: mean $=73$ range $51-89$ ) information in case records was adequate for clinical diagnosis alone in 14 of these cases, including 7 deceased (no postmortem information). 25 patients had a follow-up assessment carried out during a domicilary visit. Followup assessment was carried out using a semi-structured interview involving the Mini-Mental State Examination (MMSE) [10], an Hachinski score was calculated and a neurological examination performed. The Hamilton depression scale [16] was included for those patients with a previous diagnosis of depression. Patients were also rated according to the Clinicians Interview Based Impression of Severity scale (CIBIS plus) which is a global impression of the severity of illness at a particular point in time. Information from carers about progression of disease was also included in final clinical diagnosis.

Following review the patients were assigned a diagnostic category, according to the most likely underlying cause for their symptoms i.e. dementia of the Alzheimer's type (DAT), frontotemporal dementia (FTD), dementia of the vascular type (DVaT), and Other (other) which included depression, schizophrenia, and alcoholic dementia. Classification took into account the NINCDS/ADRDA criteria [31] for "possible" and "probable" DAT, the DSM-IV-R criteria (Diagnostic and Statistical manual of Mental Disorders 1994) [2] for vascular dementia, standard criteria for frontotemporal dementia [6], and DSM-IV-R criteria for Major Depressive Disorder and Organic Depressive Disorder.

\subsection{Image acquisition}

The patients were scanned using a SME 810 (Strichman Medical Equipment Inc.) dedicated SPECT system [46]. Slice thickness is $12 \mathrm{~mm}$, scanning time was 120 secs per slice, and slice spacing $6 \mathrm{~mm} / 8 \mathrm{~mm}$ providing an in-plane resolution of $8 \mathrm{~mm}$ and longitudinal resolution of approximately $10 \mathrm{~mm}$. The scan orientation was approximately parallel to the orbito-meatal (OM) line and head movement during the scanning procedure was minimised by subject co-operation and assisted by placing foam supports either side of the subject's head which were held in place by Velcro. For each SPECT scan a maximum dose of $500 \mathrm{MBq}^{99 \mathrm{~m}} \mathrm{Tc}$ HMPAO was injected intravenously while the patients were supine on the scanning couch under standard resting conditions of eyes closed and minimum auditory stimulus. Image acquisition began approximately 15 mins after injection. Total acquisition time was approximately 40 mins.

\subsection{Scan interpretation - visual report}

Qualitative reporting of the scans was carried out independently by two imaging specialists, both with several years experience in SPECT reporting and blinded to clinical data. The location and severity of the perfusion defects were noted and, based on the characteristic SPECT patterns described in the Introduction, scans were assigned to each of the following diagnostic categories: DAT, FTD, DVaT or Other (including normal CBF). No attempt was made to categorise LBD. In general, the reporting of scans followed the guidelines described by Holman et al. 1992 [18] and Talbot et al. 1997 [48].

\subsection{Scan interpretation - report incorporating SPM analysis}

To perform the voxel-wise statistical analysis SPM96 (Wellcome Department of Cognitive Neurology, London, UK) was run on a SPARC ULTRA 1 (SUN Microsystems Inc., Camberley, UK). This software is well documented $[3,11,12]$ for PET $\mathrm{H}_{2} \mathrm{O}$ and neuroactivation studies and consists of several steps a) image co-registration facility to correct for differences in $3 \mathrm{D}$ orientation b)spatial normalisation using a 12 point affine linear transformation into Talairach coordinate space [47] to account for differences in individual brain shape (i.e. stereotactic anatomic standardisation) c) voxelwise statistical parametric analysis based on the theory of Gaussian fields and using global mean blood flow to normalise voxel activity. The voxelwiseanalysis was used to test for the null hypothesis when comparing each individual patient scan against a group of normal CBF SPECT scans. The corresponding $\operatorname{SPM}(\mathrm{t})$ map of significantly different voxels for each patient was thresholded at $P u<0.01$ (height threshold) and $P k<0.5$ (extent threshold - corrected for multiple comparisons) for interpretation.

The control database used to perform the voxel-wise statistical analysis was constructed from 32 normal ${ }^{99 \mathrm{~m}}$ Tc HMPAO SPECT scans, from subjects aged $46-$ 68. To confirm no missed abnormalities in these scans a jack-knife analysis [33] was used to test each scan against the rest of the normal group using SPM96.

The $\operatorname{SPM}(\mathrm{t})$ maps for each patient were examined independently by the two imaging specialists together with the original SPECT scan. Based on the identification from the SPM maps of the characteristic regional abnormalities associated with each diagnostic category, a revised diagnosis was made. 
Table 1

Summary of overall assessment scores for 39 patients. All values shown are mean and range

\begin{tabular}{lccccc}
\hline no. of patients assessed & follow-up (months) & MMSE & CIBIS & HS & HDS \\
\hline 39 & $25(5-50)$ & $15.9(4-30)$ & $4.1(2-6)$ & $4.2(0-7)^{*}$ & $9(2-18)^{* *}$ \\
\hline${ }^{*}$ HS only performed on 26 patients. & & & & \\
$* *$ HDS only performed on 5 patients. \\
MMSE - mini mental state examination, CIBIS - clinicians interview based on impression of severity \\
scale, HS - Hachinski Score, HDS - Hamilton depression scale.
\end{tabular}

Table 2

The number of patients considered most likely to have a particular disease by clinical and SPECT assessments

\begin{tabular}{lcccc}
\hline & \multicolumn{4}{c}{ Number of Patients } \\
\cline { 2 - 5 } & DAT & FTD & DVaT & Other \\
\hline Clinical Assessment & 13 & 4 & 12 & 10 \\
$\begin{array}{l}\text { SPECT Visual Report } \\
\quad \text { observer A }\end{array}$ & 5 & 3 & 17 & 14 \\
$\quad$ observer B & 4 & 3 & 17 & 15 \\
SPECT Visual Report +SPM & & & & \\
$\quad$ observer A & 5 & 4 & 18 & 12 \\
$\quad$ observer B & 5 & 3 & 20 & 11 \\
SPECT Consensus Report & 6 & 3 & 18 & 12 \\
\hline
\end{tabular}

Note that the apparent agreement between observers in each diagnostic category does not indicate that these were the same subjects and hence the lower kappa values indicating disagreement in Table 4. (DAT $=$ Alzheimer's dementia, FTD $=$ Fronto-temporal dementia, DVaT $=$ Vascular dementia, other $=$ non-dementia.)

\subsection{Scan interpretation-consensus report}

Following each of these reporting methods a further review, reached by consensus between the two imaging specialists, was used to provide a final diagnosis based on imaging and SPM data against which the consensus clinical diagnosis could be compared.

\subsection{Statistical tests}

The level of agreement between imaging specialists (observers) and between the SPECT reports and clinical diagnosis was assessed using a Kappa (K)statistic [1], defined as :

$$
K=(P o-P e) /(1-P e)
$$

where $P o=$ observed proportional agreement (the total number of times the observations agreed), $P e=$ chance proportional agreement which includes a correction for the fraction of agreements expected by chance (the product of the number of choices in each category made by each observer, divided by the total number of subjects in the study). $K=1$ only when complete agreement is observed. Interpretation of the level of agreement is as follows; 0.0-0.2, slight; 0.2-0.4, fair; 0.40.6 , moderate; $0.6-0.8$, substantial, and $0.8-1.0$, almost perfect. Values below zero represent less agreement than expected by chance.

\section{Results}

\subsection{Clinical findings}

A summary of the results of clinical assessment at follow up are listed in Tables 1 and 2 .

13 patients were considered on clinical assessment to have DAT but none fulfilled the NINCDS/ADRDA criteria for "probable" disease. Similarly, none of the patients with FTD or DVaT fulfilled the relevant criteria with sufficient confidence to exclude another diagnosis. Of the 10 patients within the Other clinical category, 5 had been diagnosed with some confidence at follow-up as suffering from depression, in another 3 depression was considered the primary clinical feature, one patient had age-associated memory impairment and one was diagnosed as having schizophrenia.

\subsection{Comparison of clinical and SPECT findings}

A comparison between the clinical diagnosis and SPECT findings is shown in Table 2, while an overall assessment of consensus clinical diagnosis against consensus SPECT findings following SPM is shown in Table 3.

A significantly greater number of patients were considered to show DVaT on the SPECT than on clinical assessment while the opposite was the case with DAT. 
Table 3

Clinical and SPECT scan classification of patients using data from the neuroimaging consensus

\begin{tabular}{ccccc}
\hline & \multicolumn{4}{c}{ Imaging } \\
\cline { 2 - 5 } Clinical & DAT & FTD & DVaT & Other \\
\hline DAT & 5 & - & 2 & 6 \\
FTD & 1 & 2 & 1 & - \\
DVaT & - & 1 & 9 & 2 \\
Other & - & - & 6 & 4 \\
\hline
\end{tabular}

Of the 10 patients categorised clinically as Other, who were judged not to have dementia, 4 had a SPECT scan within the same category while 6 showed signs of cerebrovascular disease; none were considered to show DAT or FTD.

\subsection{Measures of agreement between observers and between methods}

The Kappa statistic is listed in table 4 for the following comparisons: SPECT reports between observers before and after SPM96, diagnosis between methods (clinical and SPECT) before and after SPM96 and diagnosis between methods based on consensus SPECT report after SPM96.

The agreement between the two observers for the SPECT visual reports was moderate with a Kappa of 0.57. This showed a non-significant change to 0.50 when SPM was added. The number of scans in which the two observers were in complete agreement was 28 out of $39(72 \%)$ on the visual report and 25 out of 39 $(64 \%)$ after SPM.

The agreement between the clinical assessment and the SPECT visual report was fair for both observers. SPM appeared to influence only one of the observers, causing a reduction in the Kappa value. Agreement between consensus clinical diagnosis and consensus SPECT report after SPM was fair.

The following cases illustrate the above findings. Firstly, Fig. 1 is a patient whose SPECT reports both before and after SPM96 favoured DAT, in agreement with the clinical diagnosis. Full agreement was also obtained in the patient with frontal lobe dementia shown in Fig. 2 while in those patients subsequently diagnosed as non-dementia the SPECT scans showed no significant abnormality, illustrated by the minimal, non-specific defects seen in SPM96 (Fig. 3).

Disagreements between observers were related primarily to three factors: a) Interpretation of SPECT pattern - there were differences in the allocation of SPECT patterns to pathological conditions; for example unilateral abnormalities caused uncertainty between DVaT and unilateral DAT (Fig. 4); b) Identification of abnormalities - the thresholds (both the level and extent) that each observer used to finally classify a region as abnormal varied slightly (Fig. 5) while the interpretation of high parietal defects was inconsistent (Fig. 6); c) Response to SPM - the observers reacted differently to SPM with observer 1 unlikely to change the previous visual report.

\section{Discussion}

The primary aim of this investigation was to determine whether an objective, automated method of identifying abnormal regions of cerebral perfusion, Statistical Parametric Mapping (SPM96), could aid the routine reporting of SPECT scans in individual patients whose differential diagnosis included dementia. It was postulated that a reduction in inter-observer variability would reflect an increased confidence in the visual reading of the SPECT scans thereby confirming the utility of the method. As a secondary objective the effect of the method on the level of agreement between the consensus of image reporting and the consensus clinical diagnosis determined by community based psychiatrists at an average follow-up period of 25 months was examined to determine whether there was evidence of improved diagnostic accuracy.

The study was designed to examine the influence of SPM within the routine clinical environment and was therefore carried out using a group of patients who had been referred to the Neuroimaging unit because of difficulty with diagnosis and not using a well-selected group of patients with confirmed or probable disease. Not surprisingly, the group continued to provide a diagnostic challenge even after the mean follow-up period of 25 months and most of the confident diagnoses were in those patients in whom dementia had by then been excluded. For the majority the diagnosis remained uncertain, reflecting clinical experience with this type of patient.

\subsection{Inter-observer variability and confidence of reporting}

Agreement between observers for the SPECT visual reports was only moderate, even though the general distribution of patients between the different diagnostic categories was very similar for both. This level of agreement is no worse than that found in the use of clinical rating scales such as those used for vascular 
Table 4

Measured agreements - Kappa values

\begin{tabular}{|c|c|c|c|c|c|c|}
\hline \multirow{2}{*}{$\begin{array}{c}\text { SPECT Visual } \\
\text { Report } \\
\text { Obs1 vs Obs2 }\end{array}$} & \multirow{2}{*}{$\begin{array}{c}\text { Visual Report + } \\
\text { SPM } \\
\text { Obs1 vs Obs2 }\end{array}$} & \multicolumn{2}{|c|}{$\begin{array}{c}\text { Clinical vs } \\
\text { Visual Report }\end{array}$} & \multicolumn{2}{|c|}{$\begin{array}{c}\text { Clinical vs } \\
\text { Visual Report +SPM }\end{array}$} & \multirow{2}{*}{$\begin{array}{c}\text { Clinical vs } \\
\text { Consensus } \\
\text { SPECT }\end{array}$} \\
\hline & & Obs1 & Obs 2 & Obs1 & Obs2 & \\
\hline 0.57 & 0.50 & 0.25 & 0.36 & 0.25 & 0.13 & 0.28 \\
\hline
\end{tabular}

dementia $[26,53]$ and is similar to that found in a previous SPECT study of 271 patients referred to a memory clinic [37]. The lack of false-positive diagnoses of DAT or FTD, by either observer, in the group of patients clinically diagnosed as non-dementia illustrates the predictive value of SPECT in this particular subgroup.

The ability of SPM to compare single cases with a control database has been demonstrated by the examples shown above. Several other automated methods have shown equal utility in identifying perfusion abnormalities $[4,19,33]$. This facilitation of the reporting process did not, however, translate into an improved agreement between the observers. Although non-significant, the trend was in fact in the opposite direction. This finding is of crucial importance to the use of SPECT in dementia and indicates that the interpretation of image patterns is a greater problem than the simple identification of abnormalities, and this may be especially true in those patients with difficult clinical diagnoses.

Pasquier's study [37] in a non-selected group of patients focused on the inter-observer variability in visually identifying abnormalities in specific regions of the brain and found a similar level of agreement to the present study. They did not, however, test the variability in translating those abnormalities into associated diagnostic patterns which was the essence of the present study.

\subsection{Image interpretation}

Formulating Rules - The problem of image interpretation hinges around two fundamental questions which will be now be addressed. Firstly, can unambiguous rules be formulated which relate specific levels and patterns of regional abnormality to specific diagnostic categories? Here, we are not making any assertion that the diagnostic categories are necessarily accurate pathologically. The findings of the present study suggest that such rules may not easily be achieved. SPM maps represent those regions which lie outside the chosen statistical limits of normal cerebral blood flow and, especially when no a-priori assumptions are made, small numbers of "false-positive" regions are detected [43]. Inevitably the use of SPM, as does visual reporting, requires a subjective element to resolve whether these identified regions together lie above a threshold and represent a specific pattern which would be indicative of any particular category. Perhaps the greatest variability, however, depends on how robustly the diagnostic categories are defined in terms of their imaging characteristics. One of the common examples from this study is how to classify patients with unilateral tempero-parietal abnormalities. Such unilateral defects are the sole finding in up to $25 \%$ of patients with probable $\mathrm{AD}[4,18,37]$. In the classic $\mathrm{AD}$ pattern the most affected region is Brodmann area 39 with hypoperfusion extending inferiorly to area 40 [19] yet these regions are also commonly affected by watershed ischaemia which would be a reasonable alternative diagnosis particularly in a unilateral abnormality. Similar arguments can be made regarding unilateral frontal defects which may occur in FTD [29] but may also have a vascular aetiology. The fact that such regions were often involved when the observers in the present study differed in their interpretation was clear evidence that a strategy for dealing with ambiguous regions is required to remove subjectivity and ensure consistent reporting. SPECT may have to take a lesson from general nuclear medicine in apportioning probability values [55] to a diagnosis rather than a false sense of certainty. In this respect SPM is as yet unable to contribute to this improvement.

Accuracy of SPECT - The second question addresses the issue of the accuracy of SPECT and whether a specific dementing process produces, and can therefore be recognised by, a unique perfusion pattern. For such a non-specific measurement the simplistic answer is clearly no since cerebral perfusion can be affected by a diverse range of conditions. Nevertheless different forms of dementia appear to favour different regional perfusion abnormalities allowing SPECT to be a useful adjunctive tool in the correct setting [4,14,15,18, $22,23,33,44,45,48]$. The present study showed only a fair agreement between the clinical diagnosis and the consensus SPECT diagnosis and there are a number of possible explanations for this, apart from those dis- 
cussed above. For example, studies comparing clinical diagnosis with histology have shown that clinical criteria are far from being a gold standard. The Optima study of memory and ageing, which focused on DAT, had histology in 118 cases and the diagnostic accuracy of NINCDS-ADRDA diagnosis of DAT was found to be $77 \%$ for possible DAT and only $66 \%$ for probable DAT, the latter being reduced by the low sensitivity of 49\% [23]. In another study the accepted clinical diagnostic criteria had a negative predictive value as low as $33 \%$ [34]. Clinical criteria for differentiating DAT and FTD have also been criticised when compared against histology [51]. Most studies involving histology focus on a particular category of dementia unlike the present study where patients were referred because of uncertainty in clinical diagnosis. The diagnostic accuracy of our clinical diagnoses may be poorer than that found in those studies with histology.

Publications comparing SPECT results with histology have been rare but it is enlightening to note that the studies carried out with histological confirmation [5, 23] lend greater support to the use of SPECT than those based solely on clinical criteria.

\subsection{Incidence of vascular disease}

A further factor which is likely to influence the accuracy of SPECT is the presence of multi-pathology which is commonly found at post-mortem [24]. This study has pointed in particular to the high incidence of a SPECT pattern consistent with a vascular aetiology. These features were present on SPECT in 18 of the 39 patients but only 12 of these had DVaT as the primary follow-up clinical diagnosis. The West of Scotland has one of the highest rates of cerebrovascular disease in the world and it is not surprising that a high proportion of the SPECT scans were correspondingly affected. However, the relationship between the existence of cerebrovascular disease and dementia is uncertain. Reviews in the literature $[9,25,41]$ highlight the difficulties of diagnosing vascular dementia clinically and Erkinjuntti 1997 [9] states that the main challenge is to diagnose correctly combined cases with both vascular and Alzheimer-type pathology. Most importantly, Rao and Howard [41] raise the difficulty of attributing causality and advocate defining a syndrome of global cognitive impairment associated with specific risk factors. No single feature of cerebrovascular disease is predictive of dementia and instead there appears to be a critical threshold related to the burden of the disease above which reduced cognitive function occurs [35]. In our study vascular pathology may or may not have been the major factor contributing to clinical presentation in the 18 cases detected by SPECT but it is important for clinicians to be aware of its possible presence.

\section{Conclusions}

This study has confirmed that SPM is capable of producing meaningful maps of individual patients in a routine clinical environment. However, there was no overwhelming evidence that SPM will be able to resolve many of the dilemmas presently associated with the use of SPECT in the differential diagnosis of dementia. In particular, interpretation of SPECT perfusion patterns is a bigger problem than the initial identification of abnormalities.

Differential diagnosis using cerebral perfusion SPECT involves pattern recognition. In many instances the patterns conform to the templates described in the introduction but frequently there is a degree of uncertainty due either to variations within a single pathology or to mixed pathology. The present study has demonstrated that unless an individual is directed to follow very specific rules for image interpretation there will be the potential for considerable variability in the interpretation of the same SPECT pattern. Only extensive clinical audit, preferably with feedback from histology, will provide the information necessary to refine the pattern recognition rules. It may be that objective measurements from packages like SPM will form a useful part of this iterative process. On the other hand it may be that radiochemistry developments will enable the technology to leapfrog to an altogether more fundamental level by using SPECT or PET to study directly those neurochemical deficits which are the direct markers of disease (such as with Lewy Body Dementia [54]) or are targets of therapeutic strategies.

As SPM is a robust and objective method of detecting and locating significantly abnormal regions of perfusion, it may be of value in studies seeking to quantify or classify types of cognitive and clinical deficit or could provide an objective way of monitoring the progress of a disease like dementia. A further challenge to the developers of SPM may be to extend its role to include clinical probabilities based on interaction between associated brain regions which conform to the recognised diagnostic patterns.

The uncertainties in reporting demonstrated by this study are not unique to SPECT nor to other forms of functional neuroimaging. Difficulties in setting thresh- 
olds, of recognising unique patterns of abnormality, of dealing with multi-pathologies and of separating normal ageing affect the neuropathological diagnosis of dementia with equal vigour and raise doubts even about our gold standard [28,39,40,50].

\section{References}

[1] Altman, D.G., Practical Statistics for Medical Research, Chapman and Hall, London, 1991.

[2] American Psychiatric Association, Diagnostic and StatisticalManual of Mental Disorders, American Psychiatric Association, Washington DC, 1987.

[3] J. Ashburner, P. Neelin, D.L. Collins, A. Evans and K. Friston, Incorporating prior knowledge into image registration, Neuroimage 6 (1997), 344-352.

[4] P. Bartenstein, S. Minoshima, C. Hirsch, K. Buch, F. Willoch, D. Mosch, D. Schad, M. Schwaiger and A. Kurz, Quantitative assessment of cerebral blood flow in patients with Alzheimer's disease by SPECT, Journal of Nuclear Medicine 38 (1997), 1095-1101.

[5] F.J. Bonte, M.F. Weiner, E.H. Bigio and C.L. White, Brain blood flow in the dementias: SPECT with histopathologic correlation in 54 patients, Radiology 202 (1997), 793-797.

[6] A. Brun, U. Passant, Frontal lobe degeneration of nonAlzheimer type. Structural characteristics entena and relation to other fronttemporal dementias, Acta Neurologica Scandinavia Supplement 68 (1996), 28-30.

[7] A. Burns, E. Russell and S. Page, New drugs for Alzheimer's disease, British Journal of Psychiatry 174 (1999), 476-479.

[8] R.J. Dolan, C.J. Bench, R.G. Brown, L.C. Scott, K.J. Friston and R.S. Frackowiak, Regional cerebral blood flow abnormalities in depressed patients with cognitive impairment, Journal Neurology, Neurosurgery and Psychiatry 55 (1992), 768-773.

[9] T. Erkinjuntti, Vascular dementia: challenge of clinical diagnosis, International Psychogeriatrica 9(1) (1997), 51-58.

[10] M.F. Folstein, S.E. Folstein and P.R. McHugh, Mini-mental state. A practical method for grading the cognitive state of patients for the clinician, Journal of Psychiatric Research 12 (1975), 189-198.

[11] Friston, K.J., Holmes, A.P., Poline, J.B., Price, C.J. and Frith, C.D., Detecting activations in PET and fMRI: levels of inference and power, Neuroimage 4 (1996), 223-235.

[12] Friston, K.J., Holmes, A.P., Worsley, K.J., Poline, J.B., Frith, C.D. and Frackowiak, R.S.J., Statistical Parametric Mapping: A general linear approach, Human Brain Mapping 12 (1995), 189-210.

[13] D.S. Geldmacher, P.J. Whitehouse and Jr., Differential diagnosis of Alzheimer's disease, Neurology 48 (1997), S2-S9.

[14] H.G. Gemmell, P.F. Sharp, J.A. Besson, J.R. Crawford, K.P. Ebmeier, J. Davidson and F.W. Smith, Differential diagnosis in dementia using the cerebral blood flow agent ${ }^{99 \mathrm{~m}}$ Tc HM-PAO: a SPECT study, Journal of Computer Assisted Tomography 11 (1987), 398-402.

[15] H.G. Gemmell, P.F. Sharp, F.W. Smith, J.A. Besson, K.P. Ebmeier, J. Davidson, N.T. Evans, D. Roeda, R. Newton and J.R. Mallard, Cerebral blood flow measured by SPECT as a diagnostic tool in the study of dementia, Psychiatry Research 29 (1989), 327-329.

[16] M. Hamilton, Development of a rating scale for primary depressive illness, British Journal of Social and Clinical Psychology 6 (1967), 278-296.
[17] A. Heyman, G.G. Fillenbaum, K.A. Welsh-Bohmer, M. Gearing, S.S. Mirra, R.C. Mohs, B.L. Peterson and C.F. Pieper, Cerebral infarcts in patients with autopsy-proven Alzheimer's disease: CERAD, part XVIII. Consortium to Establish a Registry for Alzheimer's Disease [published erratum appears in Neurology 1998 Dec; 51(6): 1809], Neurology 51 (1998), 159-162.

[18] B.L. Holman, K.A. Johnson, B. Gerada, P.A. Carvalho and A. Satlin, The scintigraphic appearance of Alzheimer's disease: a prospective study using technetium-99m-HMPAO SPECT [published erratum appears in J Nucl Med 1992 Apr; 33(4): 484] [see comments], Journal of Nuclear Medicine 33 (1992), $181-185$.

[19] A.S. Houston, P.M. Kemp and M.A. Macleod, A method for assessing the significance of abnormalities in HMPAO brain SPECT images, Journal Nuclear Medicine 35 (1994), 239244.

[20] Ibanez, V., Pietrini, P., Alexander, G.E., Furey, M.L., Teichberg, D., Rajapakse, J.C., Rapoport, S.I., Schapiro, M.B. and Horwitz, B., Regional glucose metabolic abnormalities are not the result of atrophy in Alzheimer's disease, Neurology $\mathbf{5 0}$ (1998), 1585-1593.

[21] M. Jackson and J. Lowe, The new neuropathology of degenerative frontotemporal dementias, Acta Neuropathology (Berl) 91 (1996), 127-134.

[22] W.J. Jagust, K.A. Johnson and B.L. Holman, SPECT perfusion imaging in the diagnosis of dementia, Journal of Neuroimaging 5(1) (1995), S45-S52.

[23] K.A. Jobst, L.P. Barnetson and B.J. Shepstone, Accurate prediction of histologically confirmed Alzheimer's disease and the differential diagnosis of dementia: the use of NINCDSADRDA and DSM- III-R criteria, SPECT, X-rayCT, and Apo E4 in medial temporal lobe dementias. Oxford Project to Investigate Memory and Aging [corrected and republished article originally printed in Int Psychogeriatr 1997; 9 Suppl1: 191222; discussion 247-252], International Psychogeriatrica 10 (1998), 271-302.

[24] A. Lim, D. Tsuang, W. Kukull, D. Nochlin, J. Leverenz, W. McCormick, J. Bowen, L. Teri, J. Thompson, E.R. Peskind, M. Raskind and E.B. Larson, Clinico-neuropathological correlation of Alzheimer's disease in a community-based case series, Journal of American Geriatric Society 47 (1999), 564-569.

[25] C. Loeb and J.S. Meyer, Vascular dementia: still a debatable entity? Journal of Neurological Sciences 143 (1996), 31-40.

[26] Lopez, O.L., Larumbe, M.R., Becker, J.T., Rezek, D., Rosen, J., Klunk, W. and DeKosky, S.T., Reliability of NINDSAIREN clinical criteria for the diagnosis of vascular dementia, Neurology 44 (1994), 1240-1245.

[27] J. Lowe, Establishing a pathological diagnosis in degenerative dementias, Brain Pathology 8 (1998), 403-406.

[28] I.R. Mackenzie and D.G. Munoz, Nonsteroidal antiinflammatory drug use and Alzheimer-type pathology in aging, Neurology 50 (1998), 986-990.

[29] D.M. Mann, P.W. South, J.S. Snowden and D. Neary, Dementia of frontal lobe type: neuropathology and immunohistochemistry, Journal of Neurology, Neurosurgery and Psychiatry 56 (1993), 605-614.

[30] I.G. McKeith, D. Galasko, K. Kosaka, E.K. Perry, D.W. Dickson, L.A. Hansen, D.P. Salmon, J. Lowe, S.S. Mirra, E.J. Byrne, G. Lennox, N.P. Quinn, J.A. Edwardson, P.G. Ince, C. Bergeron, A. Burns, B.L. Miller, S. Lovestone, D. Collerton, E.N. Jansen, C. Ballard, R.A. de Vos, G.K. Wilcock, K.A. Jellinger and R.H. Perry, Consensus guidelines for the clinical and pathologic diagnosis of dementia with Lewy bodies 
(DLB): report of the consortium on DLBinternational workshop, Neurology 47 (1996), 1113-1124.

[31] G. McKhann, D. Drachman, M. Folstein, R. Katzman, D. Price and E.M. Stadlan, Clinical diagnosis of Alzheimer's disease: report of the NINCDS-ADRDA WorkGroup under the auspices of Department of Health and Human Services Task Force on Alzheimer's Disease, Neurology 34 (1984), 939-944.

[32] B.L. Miller, C. Ikonte, M. Ponton, M. Levy, K. Boone, A. Darby, N. Berman, I. Mena and J.L. Cummings, A study of the Lund-Manchester research criteria for frontotemporal dementia: clinical and single-photon emission CT correlations, Neurology 48 (1997), 937-942.

[33] S. Minoshima, K.A. Frey, R.A. Koeppe, N.L. Foster and D.E. Kuhl, A diagnostic approach in Alzheimer's disease using three-dimensional stereotactic surface projections of fluorine18-FDG PET, Journal of Nuclear Medicine 36 (1995), 12381248.

[34] Z. Nagy, M.M. Esiri, N.J. Hindley, C. Joachim, J.H. Morris, E.M. King, B.Mc. Donald, S. Litchfield, L. Barnetson, K.A. Jobst and A.D. Smith, Accuracy of clinical operational diagnostic criteria for Alzheimer's disease in relation to different pathological diagnostic protocols, Dementia Geriatric Cognitive Disorders 9 (1998), 219-226.

[35] O'Brien, M.D., How does cerebrovascular disease cause dementia? Dementia 5 (1994), 133-136.

[36] L. Parnetti, D.T. Lowenthal, O. Presciutti, G.P. Pelliccioli, R. Palumbo, G. Gobbi, P. Chiarini, B. Palumbo, R. Tarducci and U. Senin, 1H-MRS, MRI-based hippocampal volumetry, and ${ }^{99 \mathrm{~m}}$ Tc-HMPAO-SPECT in normal aging, age-associated memory impairment, and probable Alzheimer's disease [see comments], Journal of American GeriatricSociety 44 (1996), 133-138.

[37] F. Pasquier, I. Lavenu, F. Lebert, B. Jacob, M. Steinling and H. Petit, The use of SPECT in a multidisciplinary memory clinic, Dementia Geriatric CognitiveDisorders 8 (1997), 85-91.

[38] F. Pasquier and D. Leys, Why are stroke patients prone to develop dementia? Journal of Neurology 244 (1997), 135142.

[39] J.M. Powers, Diagnostic criteria for the neuropathologic assessment of Alzheimer's disease, Neurobiology and Aging $\mathbf{1 8}$ (1997), S53-S54.

[40] Price, J.L., Diagnostic criteria for Alzheimer's disease, Neurobiology and Aging 18 (1997), S67-S70.

[41] R. Rao and R. Howard, Vascular dementia: dead or alive? International Journal of Geriatric Psychiatry 13 (1998), 277284.

[42] G.C. Roman, T.K. Tatemichi, T. Erkinjuntti, J.L. Cummings, J.C. Masdeu, J.H. Garcia, L. Amaducci, J.M. Orgogozo, A. Brun and A. Hofman, X. Vascular dementia: diagnostic criteria for research studies. Report of the NINDS-AIREN International Workshop [see comments], Neurology 43 (1993),
250-260.

[43] M. Signorini, E. Paulesu, K. Friston, D. Perani, A. Colleluori, G. Lucignani, F. Grassi, V. Bettinardi, R.S.J. Frackowiak and F. Fazio, Rapid assessment of regional cerebral metabolic abnormalities in single subjects with quantitative and nonquantitative [18F]FDG PET: A clinical validation of statistical parametric mapping, Neuroimage 9 (1999), 63-80.

[44] F.W. Smith, J.A. Besson, H.G. Gemmell and P.F. Sharp, The use of technetium-99m-HM-PAO in the assessment of patients with dementia and other neuropsychiatric conditions, Journal of Cerebral Blood Flow and Metabolism 8 (1988), S116-S122.

[45] F.W. Smith, H.G. Gemmell and P.F. Sharp, The use of 99TcmHM-PAO for the diagnosis of dementia, Nuclear Medicine Communications 8 (1987), 525-533.

[46] Stoddart, H.A. and Stoddart, H.F., New multi-dimensional reconstructions for the 12-detector, scanned focal point, singlephoton tomograph, Physics inMedicine and Biology 37 (1992), 579-586.

[47] Talairach, J. and Tournoux, P., Co-planar stereotaxic atlas of the human brain, Thieme Medical Publishers, New York, 1988.

[48] P.R. Talbot, J.J. Lloyd, J.S. Snowden, D. Neary and H.J. Testa, A clinical role for ${ }^{99 \mathrm{~m}} \mathrm{Tc}$-HMPAO SPECT in the investigation of dementia? Journal of Neurology, Neurosurgery and Psychiatry 64 (1998), 306-313.

[49] P. Taylor, Invited review: computer aids for decision-making in diagnostic radiology - a literature review, British Journal of Radiology 68 (1995), 945-957.

[50] J.Q. Trojanowski, C.M. Clark, M.L. Schmidt, S.E. Arnold and V.M. Lee, Strategies for improving the postmortem neuropathological diagnosis of Alzheimer's disease, Neurobiology and Aging 18 (1997), S75-S79.

[51] A.R. Varma, J.S. Snowden, J.J. Lloyd, P.R. Talbot, D.M. Mann and D. Neary, Evaluation of the NINCDS-ADRDA criteria in the differentiation of Alzheimer's disease and frontotemporal dementia, Journal Neurology, Neurosurgery and Psychiatry 66 (1999), 184-188.

[52] A.R. Varma, P.R. Talbot, J.S. Snowden, J.J. Lloyd, H.J. Testa and D. Neary, A $^{99 \mathrm{~m}}$ Tc-HMPAO single-photon emission computed tomography study of Lewy body disease, Journal of Neurology 244 (1997), 349-359.

[53] Verhey, F.R., Lodder, J., Rozendaal, N. and Jolles, J., Comparison of seven sets of criteria used for the diagnosis of vascular dementia, Neuroepidemiology 15 (1996), 166-172.

[54] Walker, Z., Costa, D.C., Ince, P., McKeith, I.G. and Katona, C.L., In-vivo demonstration of dopaminergic degeneration in dementia with Lewy bodies, Lancet 354 (1999), 646-647.

[55] Worsley, D.F. and Alavi, A., Comprehensive analysis of the results of the PIOPED Study, Prospective Investigation of Pulmonary Embolism Diagnosis Study, Journal of Nuclear Medicine 36 (1995), 2380-2387. 

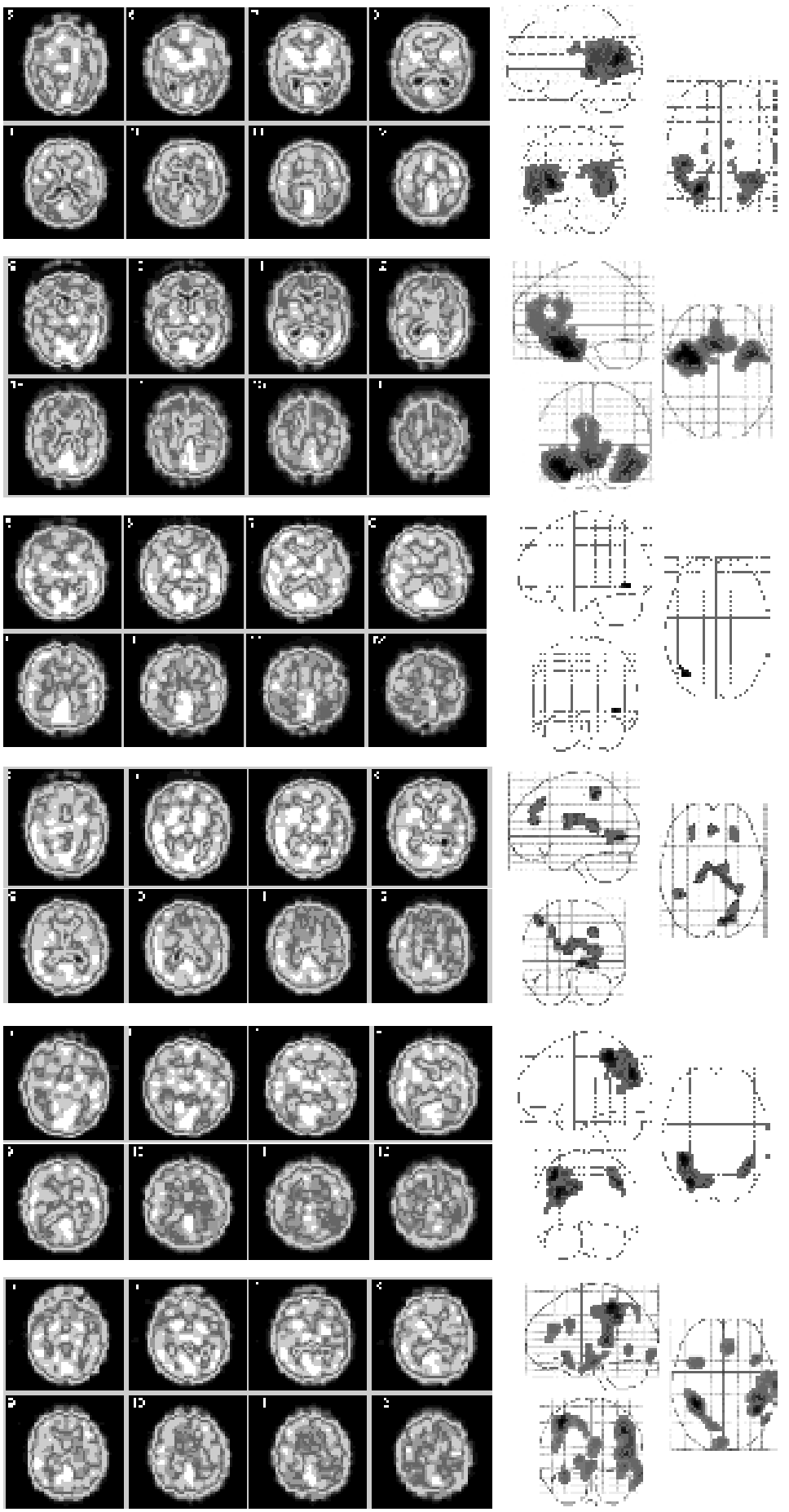

(a)
The diagnosis of DAT for this patient was agreed by both clinical and imaging observers.

The diagnosis of FTD for this patient was agreed by both clinical and imaging observers.

The diagnosis of non-dementia for this patient was agreed by both clinical and imaging observers.

This patient was given a DAT/DVaT (mixed pathology) diagnosis from observer 1 and a DVaT diagnosis from observer 2. Slight variation in the thresholds used by each observer to identify abnormalities resulted in difference of diagnosis. The $\operatorname{SPM}(\mathrm{t})$ map also shows evidence of enlarged ventricles.

This patient was given a non dementia diagnosis fromobserver 1 and a DAT diagnosis from observer 2. Unilateral abnormalities caused disagreement between observers.

This patient was given a DVaT diagnosis from observer 1 and a DAT diagnosis from observer 2. Interpretation of high parietal defects differed between observers.

Figures 1-6a show Tc99m HMPAO SPECT scans of 8 (6 mm thick) axial slices, above and below the basal ganglia. Figures $1-6 \mathrm{~b}$ show the corresponding SPM(t) mat of the maximum intensity projections (MIP) of the voxels surviving a significance threshold of Pu $<0.01$ and $\mathrm{Pk}<$ 0.5 (corrected for multiple comparisons). 


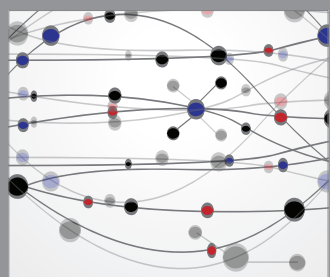

The Scientific World Journal
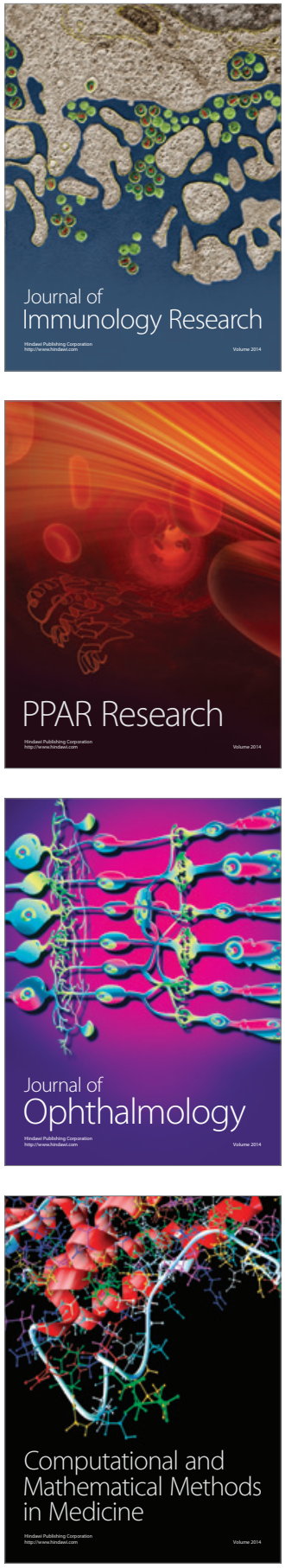

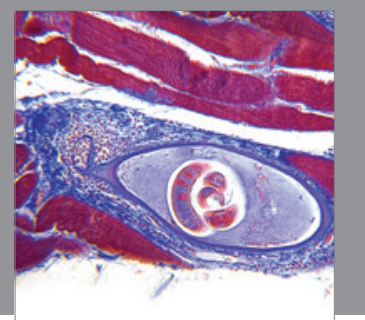

Gastroenterology

Research and Practice
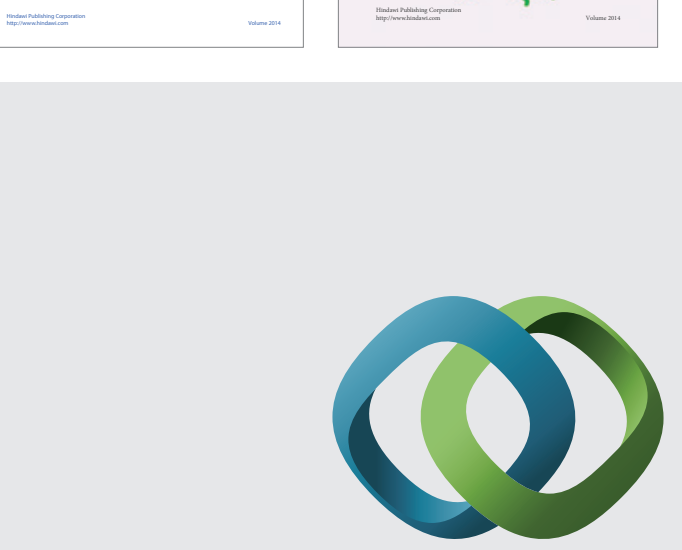

\section{Hindawi}

Submit your manuscripts at

http://www.hindawi.com
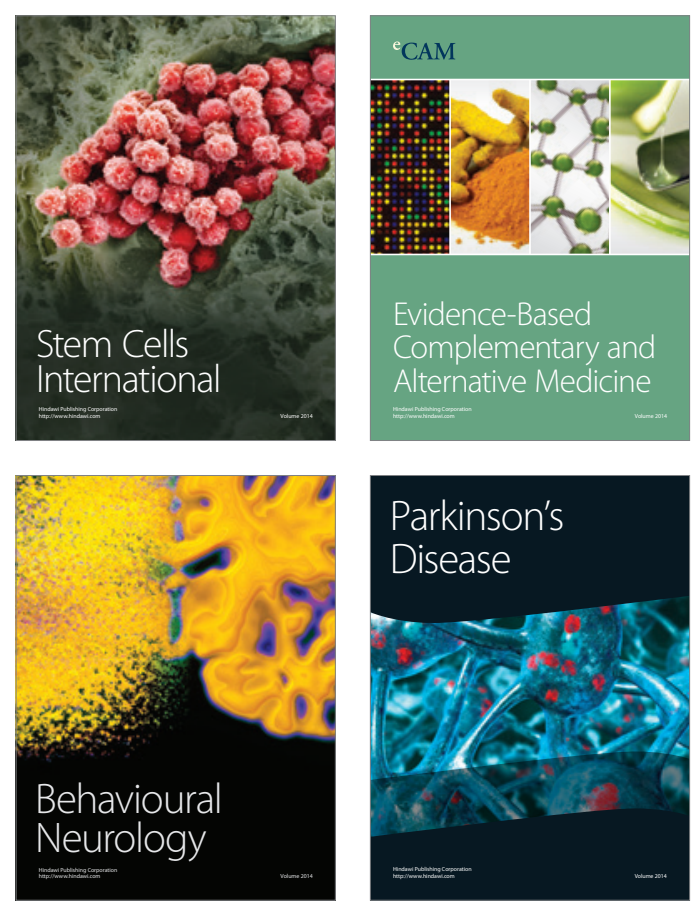

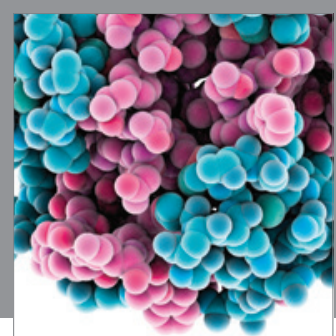

Journal of
Diabetes Research

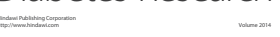

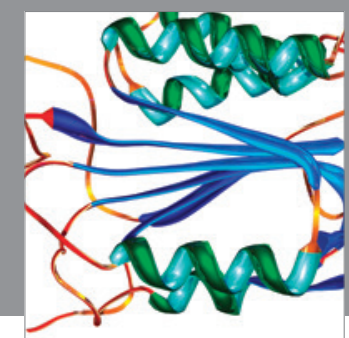

Disease Markers
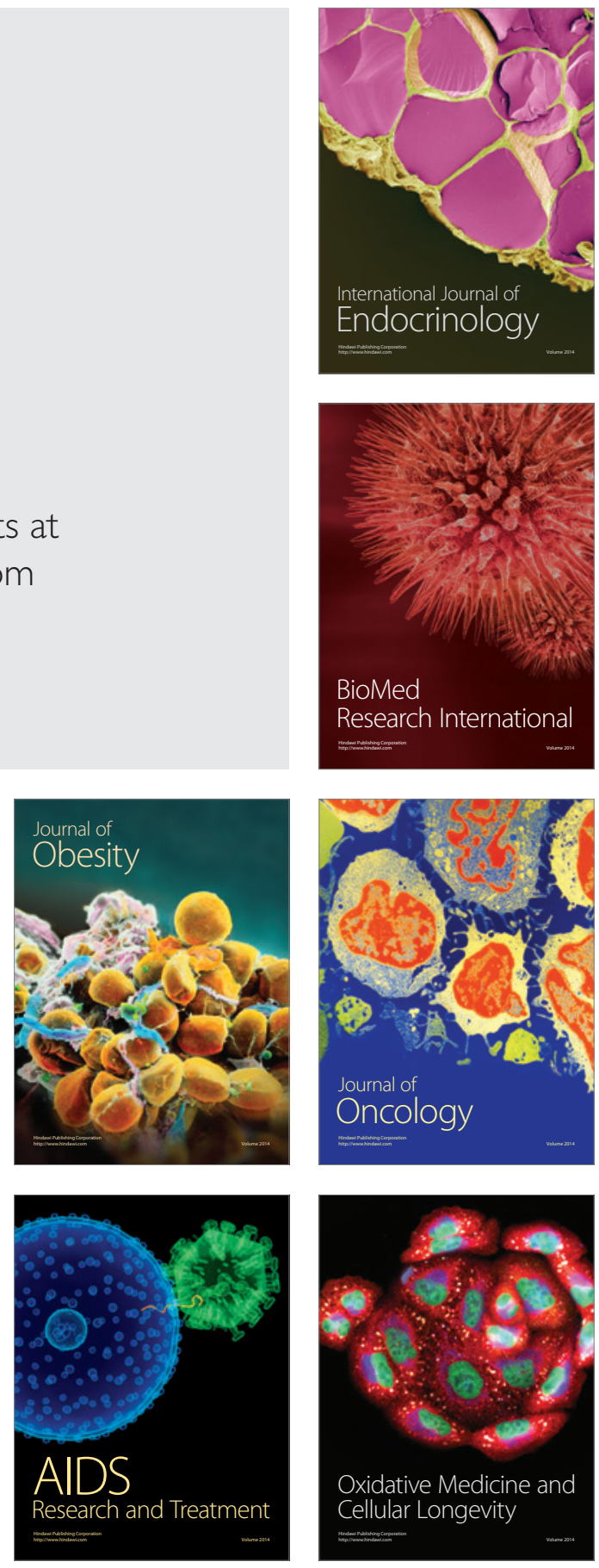